Genre et filiation : pratiques et représentations

\title{
Filiations mexicaines au temps de la Révolution
}

\section{Nicolas BALUTET}

\section{OpenEdition}

\section{Journals}

Édition électronique

URL : http://journals.openedition.org/transtexts/483

DOI : $10.4000 /$ transtexts.483

ISSN : 2105-2549

\section{Éditeur}

Gregory B. Lee

\section{Référence électronique}

Nicolas BALUTET, «Filiations mexicaines au temps de la Révolution», Transtext(e)s Transcultures 跨文 本跨文化 [En ligne], 8 | 2013, mis en ligne le 02 décembre 2013, consulté le 30 avril 2019. URL : http:// journals.openedition.org/transtexts/483 ; DOI : 10.4000/transtexts.483 


\section{Transtext(e)s Transcultures 跨文本跨 文化}

Journal of Global Cultural Studies

$8 \mid 2013$ :

Genre et filiation : pratiques et représentations

Récits de filiation et (ré)écriture de soi

\section{Filiations mexicaines au temps de la Révolution}

\section{NICOLAS BALUTET}

\section{Résumé}

Como agua para chocolate (1989) de Laura Esquivel, Por debajo del agua (2002) de Fernando Zamora et Por si no te vuelvo a ver (2007) de Laura Martínez-Belli sont trois romans qui partagent de nombreux points communs : un fond mélodramatique, un ancrage spatial et historique, l'importance des soldaderas, des prostituées et des servantes indiennes, un positionnement clairement féministe, des modalités narratives identiques, etc. Ces textes placent également au cœur de leur récit un sujet qui a été l'un des plus débattus en France au cours des premiers mois de l'année 2013 : la filiation. Pour les tenants d'une conception traditionnelle de la famille, la filiation ne peut reposer que sur le mariage entre un homme et une femme et sur un lien biologique. Cependant, c'est oublier bien vite non seulement les mutations profondes qu'a connues la famille depuis le milieu des années 60 mais également le sens même du terme " filiation ". En s'appuyant sur les romans cités, cet article entend montrer que la filiation n'est pas synonyme de lien biologique et qu'il ne faut pas confondre l'engendrement (le fait de concevoir et de donner naissance à un enfant) et l'engagement (le projet parental autour de l'enfant).

\section{Texte intégral}

La littérature mexicaine et, plus généralement, l'ensemble de la littérature mondiale, regorge de représentations de parents au comportement indigne. Un des exemples les plus frappants -c'est l'adjectif qui convient- se trouve dans le best-seller de Laura Esquivel (1950-), Como agua para chocolate (1989) ${ }^{1}$ qui, à 
l'heure actuelle, se serait vendu à pas moins de 4,5 millions d'exemplaires dans le monde. ${ }^{2}$ Dans ce roman, connu de tout hispaniste, le personnage d'Elena est constamment associé au qualificatif mamá, « maman ». Pourtant, son attitude contrevient à l'idée que l'on se fait généralement d'une mère et des parents. Apprenant que sa fille Gertrudis travaille dans une maison close, Mamá Elena est capable, par exemple, de détruire son acte de naissance afin de l'effacer de la généalogie de la famille et d'ordonner qu'on ne mentionne plus jamais son nom. ${ }^{3}$ Elle fait preuve, par ailleurs, non seulement de violence verbale mais aussi physique : elle donne à sa dernière fille Tita, de manière graduelle, " quantité de gifles » ${ }^{4}$ parce que Tita ne veut pas l'appeler «mami », une version plus douce encore que " maman », " une gifle monumentale [qui l'envoie] rouler à terre $»^{5}$, « une formidable raclée [...], comme jamais auparavant ou par la suite $»^{6}$. Enfin, quand Tita ose lui tenir tête, Mamá Elena s'empare d'une cuiller en bois et la frappe au visage. ${ }^{7}$

2 La description de la mère qui est proposée tout au long du roman la place sous le signe de la destruction (champ lexical de la coupe, du tranchage, de la brisure) dont le meilleur exemple est l'extrait suivant :

\begin{abstract}
Mamá Elena avait l'art de couper la pastèque. Elle enfonçait la pointe d'un couteau très effilé dans la partie verte sans toucher le cœur du fruit. Elle pratiquait ensuite une série d'incisions d'une telle précision que lorsque, à la fin, elle saisissait la pastèque pour la frapper sur une pierre - une seule fois et au bon endroit -, l'écorce s'ouvrait magiquement, comme des pétales de fleur, autour du cour intact. C'est sûr : pour tout ce qui est de couper, diviser, démembrer, ravager, séparer, sectionner, bouleverser ou détruire, Mamá Elena était une experte. ${ }^{8}$
\end{abstract}

La répétition accumulative des verbes à l'infinitif, que l'on retrouve au dernier chapitre où il est rapporté que Mamá Elena « non contente de casser d'innombrables sacs de noix pendant des jours, [...] y prenait plaisir : écraser, ouvrir et décortiquer, c'étaient ses activités favorites ${ }^{9}$, renforce le bruit du couteau et accentue la capacité destructrice du personnage qui ressemble ici à un prêtre aztèque effectuant un sacrifice coronarien. Il est intéressant de noter que cet épisode est décrit comme un acte de pénétration qui permet au melon d'eau de s'ouvrir " comme des pétales de fleur », plaçant la destruction sous le sceau sexuel. ${ }^{10}$ Mamá Elena ne se contente pas de détruire dans la sphère alimentaire, elle anéantit aussi ceux qui l'entourent comme en témoignent les propos explicites de Tita, condamnée par sa mère à rester célibataire et à s'occuper d'elle jusqu'à sa mort : "Pour elle, Mamá Elena avait fait une exception, elle avait commencé à la tuer dès l'enfance, à petit feu ». ${ }^{11}$ Dans un essai, Laura Esquivel elle-même a comparé Mamá Elena à la déesse aztèque Coatlicue dont tous les visiteurs du Musée National d'Anthropologie de Mexico n'ont pu que remarquer l'aspect effrayant. Le personnage apparaît comme "l'image d'une mère sorcière maintenant les mâchoires de ses enfants [...] l'image de la malnutrition spirituelle, de la censure verbale, du silence de la poésie, de l'esprit enchaîné. En quelques mots, [...] l'image de la véritable fille de... Coatlicue, la dévoratrice $» .^{12}$

4 Deux autres romans plus récents, Por debajo del agua (2002) de Fernando Zamora (1969-) ${ }^{13}$ et Por si no te vuelvo a ver (2007) de Laura Martínez-Belli $\left(1975^{-}\right)^{14}$, présentent également des personnages qui se soustraient à leur " exercice de parentalité », entendu suivant Hervé Hamon dans le sens des droits et des devoirs afférents au fait d'être parent. ${ }^{15}$ Dans Por debajo del agua, 
la mère Isabel abandonne ses quatre enfants pour s'enfuir à Ciudad Juárez " avec un homme à l'identité inconnue ${ }^{16}$, plongeant toute sa famille dans un profond désespoir et son mari dans l'alcoolisme. ${ }^{17} \mathrm{Ce}$ dernier, quelques années plus tard, finira lui aussi par abandonner ses enfants dont certains sont encore mineurs, les « congédiant " selon ses propres mots ${ }^{18}$, avant de mettre fin à ses jours. L'histoire est plus cruelle encore dans Por si no te vuelvo a ver qui s'ouvre sur l'abandon de Mercedes, alors âgée d'à peine 13 ans, laissée au sein d'une maison close de Veracruz, El Gallo Tuerto, "Le coq borgne », un nom presque oxymoronique puisqu'il suggère une forte virilité (à travers le coq) quelque peu malmenée par la perte d'un attribut important (un œil qui peut être ici une allusion à un testicule). Le texte suggère que la mère a vendu sa fille contre de l'argent, la dévaluant en faisant de son corps un objet de consommation, d'autant plus prisé qu'il est vierge et à peine pubère. L'histoire va se répéter quelques années plus tard avec l'abandon du fils de Mercedes, Gilberto, mais, dans ce cas précis, il apparaît que la mère agit ainsi dans l'intérêt supérieur de son enfant. ${ }^{19}$ Les commentaires d'amour maternel sont légions dans le roman, à l'instar des nombreux moments de doute et de remords de Mercedes ${ }^{20}$ qui n'est pas sans rappeler La Llorona, cette figure légendaire mexicaine désignant une belle femme souvent indienne, mère d'un ou de plusieurs fils illégitimes qui, après avoir été abandonnée par son amant, prise de folie, décide de noyer sa progéniture dans une rivière ou bien de la manger. Suite à ce crime et prise de remords, elle erre toutes les nuits à la recherche de ses enfants défunts, en poussant des cris de lamentations. ${ }^{21}$ L'abandon de Gilberto relève davantage, me semble-t-il, du don d'enfant, fort bien étudié pour la France par Agnès Fine. ${ }^{22}$

$5 \quad$ Le rapprochement de ces trois récits mexicains n'est pas le fruit du hasard. Ils partagent en effet de nombreux points communs : un fond mélodramatique, un ancrage spatial et historique -le Mexique de la fin du XIXe siècle à la première moitié du XXe avec une intrigue principale centrée sur la période révolutionnaire 1910-1920 ${ }^{23}$-, l'importance des servantes indiennes, des prostituées et des soldaderas, ces femmes qui accompagnaient les troupes révolutionnaires pour combattre ou pour se charger de l'intendance ou de l'infirmerie, un positionnement clairement féministe chez les deux écrivaines ${ }^{24}$, des narrateurs extra-hétéro-diégétiques, etc. Ces romans placent également au cœur de leur récit un sujet qui me semble avoir été l'un des plus débattus en France au cours de la première moitié de l'année : la filiation. L'année 2013 aura été marquée, en effet, par un événement majeur dans notre pays : l'ouverture du mariage et de l'adoption aux couples homosexuels. La France a ainsi rejoint, tardivement certes, la liste des pays et états où ces mêmes droits sont désormais reconnus. Lors des débats, outre l'homophobie de certains intervenants, « honteuse » au début des débats puis clairement affichée, il est apparu évident que les détracteurs du « mariage pour tous » qui, une quinzaine d'années auparavant, luttaient déjà contre le Pacte civil de solidarité, s'attelaient à dénoncer, outre l'union de personnes du même sexe, le dérèglement de la filiation qui, selon eux, en découlerait. Pour ces tenants d'une conception traditionnelle de la famille, la filiation semble ne reposer, en effet, que sur le mariage entre un homme et une femme et sur un lien biologique.

6 Cette conception, récente et propre à la pensée occidentale ${ }^{25}$, va pourtant à l'encontre du sens même de la notion de filiation qui, selon Anne Lefebre- 
Teillard dans le Dictionnaire de la culture juridique, est « un concept d'essence politique au sens propre du terme. C'est pourquoi depuis l'Antiquité la filiation est définie comme un lien de droit qui ne se confond pas avec le lien biologique $»{ }^{26}$ Les règles relatives à la filiation sont d'ordre culturel et ne relèvent pas de la nature :

Un enfant à la naissance est désaffilié ou, plus exactement, non affilié. C'est sa reconnaissance juridique qui signe son affiliation [...] il ne suffit pas que l'événement de la naissance se produise pour qu'ipso facto il entraîne des effets juridiques, à savoir l'établissement du lien de filiation. En réalité, une manifestation de la volonté est nécessaire pour établir ce lien. La tradition juridique moderne porte ainsi en elle la déconnexion entre le biologique et la filiation. ${ }^{27}$

Le fait que nous soyons les héritiers d'un modèle matrimonial fondé sur le principe d'une complémentarité hiérarchique des sexes, finalement assez récent ${ }^{28}$, explique peut-être en partie que l'argument biologique continue de s'imposer dans l'esprit de beaucoup de gens alors même que ce modèle a été totalement bouleversé depuis le milieu des années 60 avec l'augmentation des naissances naturelles, les divorces, la monoparentalité, l'homoparentalité, les familles recomposées, etc. Il existe aujourd'hui un véritable kaléidoscope de familles. Outre le simple fait qu'être le père ou la mère biologique ne garantit nullement un bon exercice de la parentalité comme en témoignent les trois romans mexicains, on voit bien à travers l'adoption, qui est un lien de filiation sans procréation et en dehors des liens du sang, les limites de l'argument biologique. Il en va de même avec ce que l'on appelle la «présomption de paternité ». Que ce soit en France ou au Mexique, où le système juridique repose en grande partie sur le Code Napoléon, le mariage a été pensé comme une institution reposant sur une hypothèse : la présomption de paternité - le fameux adage latin Pater is est quem nuptiae demonstrant -, c'est-à-dire la reconnaissance anticipée de futurs enfants nés de l'union d'une femme et de son mari, forcément le géniteur, qui s'engage à les nourrir, les élever, leur transmettre le patrimoine, etc. ${ }^{29}$ Or, cette idée s'est considérablement affaiblie, avec notamment, en France et au Mexique, l'effacement de la distinction entre enfants naturels (nés hors mariage) et légitimes (en mariage) et la réalité dévoilée par les résultats des tests de paternité très en vogue actuellement dans les pays anglo-saxons. ${ }^{30}$ Dans Como agua para chocolate par exemple, le père de Gertrudis -la deuxième fille- n'est pas Monsieur de La Garza mais un jeune métis dont était éperdument amoureuse Mamá Elena, double transgression dans un Mexique qui loue les qualités des Blancs au détriment de celles des Indiens ou des Noirs. Décidée à s'enfuir avec le jeune homme, José Treviño, la mère de famille dut se résigner à rester aux côtés de son mari après avoir aperçu un inconnu tapi dans l'ombre se jeter sur son amant et le tuer..$^{31} \mathrm{Il}$ est important de ne pas confondre l'engendrement, " le fait de concevoir et de donner naissance à un enfant $»^{32}$ et l'engagement : l'engagement d'un homme, d'une femme, d'un couple hétéro- ou homosexuel à prendre un enfant pour fils ou fille, qu'il soit ou non son géniteur. ${ }^{33}$ Le mariage est aujourd'hui une institution reconnaissant le lien unissant un couple et l'éventuelle filiation qui en découle repose davantage sur la réalité du projet parental autour de l'enfant que sur la p/maternité biologique.

$8 \quad$ Por si no te vuelvo a ver et Como agua para chocolate pourraient, de prime abord, illustrer cette dissociation entre la filiation et le biologique. Dans le 
roman de Laura Esquivel, c'est la cuisinière otomi Nacha qui apparaît comme la « vraie » mère de Tita. Elle s'occupe de l'alimenter dès la naissance ${ }^{34}$, lui donnant à boire du thé et de l'atole, une boisson à base de maïs, puis forme son palais à toutes sortes de mets dont des plats que mangeaient ses ancêtres indiens. ${ }^{35}$ Dans les souvenirs de Tita, Nacha est ainsi toujours associée à la nourriture $^{36}$. On pourrait presque la qualifier de "Déesse Mère » tant la cuisinière rappelle, selon les propres mots de Laura Esquivel, "l'image de Tonantzin-Guadalupe, la bonne mère, la protectrice, celle qui nourrit, celle qui, grâce aux aliments, libère l'esprit, la sensualité et tous les désirs accumulés en silence ». ${ }^{37}$ Mais Nacha n'est pas seulement une pourvoyeuse d'aliments, elle est également une complice de jeux ${ }^{38}$, économisant son maigre salaire pour acheter un zootrope à Tita ${ }^{39}$, une confidente qui apaise les peines ${ }^{40}$, toujours présente, malgré la mort, lors des moments clés de la vie de sa "petite.$^{41}$ Enfin, lors du premier et difficile accouchement de Rosaura, la fille aînée de la famille, c'est grâce à l'esprit de Nacha que Tita peut sauver le bébé et sa mère ${ }^{42}$ :

Tita, agenouillée devant Rosaura, au comble du désespoir, supplia Nacha de lui apporter quelques lumières. Puisqu'elle lui dictait des recettes de cuisine, Nacha pouvait également l'aider maintenant ! Il fallait bien que quelqu'un prête assistance à Rosaura depuis l'au-delà, puisque ceux d'ici-bas en étaient incapables.

Elle ne savait pas combien de temps elle avait prié à genoux. Mais quand enfin elle décolla ses paupières, le tunnel obscur se transforma soudain en un fleuve rouge, en un volcan impétueux, il y eut un déchirement de papier. La chair de sa sœur s'ouvrait à la vie. [...]

\begin{abstract}
Elle prit l'enfant dans ses mains, le tendit à Rosaura et toutes deux pleurèrent au-dessus de lui. Ensuite, elle n'eut qu'à suivre les instructions que Nacha lui chuchotait à l'oreille. Elle exécuta avec précision les gestes nécessaires : couper le cordon ombilical à l'endroit et au moment précis, laver le corps de l'enfant avec de l'huile d'amande douce, lui bander le nombril et l'habiller. Elle enfila d'abord le maillot et la chemise, puis posa la bande sur le nombril, lui mit la couche en coton, une deuxième couche, la flanelle sur les jambes, la brassière, les chaussettes et les chaussons. Enfin elle lui croisa les mains sur la poitrine et le recouvrit d'une couverture en peluche pour l'empêcher de se gratter la figure. Lorsque Mamá Elena et Chencha revinrent le soir, accompagnées de Lobo, elles admirèrent le travail [...] Désormais, on ne s'inquiétait que de la santé de Rosaura, encore très fragile et toute enflée. Ce fut alors qu'on découvrit les risques qu'elle avait courus pendant son accouchement : selon le médecin, Rosaura avait souffert d'une attaque d'éclampsie qui aurait pu la tuer. Il se montra très surpris de l'aplomb et de l'esprit de décision de Tita dans des conditions aussi difficiles. ${ }^{43}$
\end{abstract}

De son côté, Tita occupe également une fonction de mère de substitution auprès de son neveu Roberto qu'elle allaite de manière surnaturelle alors qu'elle est toujours vierge ${ }^{44}$, puis de sa nièce Esperanza qu'elle alimente, comme Nacha l'avait fait avec elle, avec des tisanes et des atoles. ${ }^{45}$

Dans le roman de Laura Martínez-Belli, Gilberto est élevé, jusqu'à ses six ans, avec beaucoup d'amour par les neufs prostituées du Gallo Tuerto « qui le choyaient et le protégeaient comme s'il s'agissait d'un moineau s'étant cogné comme une porte en verre. Le soir, elles lui racontaient des histoires, lui apprenaient à s'habiller, chatouillaient son ventre et lui permettaient de dessiner autant qu'il le désirait sur les murs de la cuisine ».46 Peut-être voient-elles en lui l'enfant qui leur est interdit d'avoir pour pouvoir continuer à 
travailler et survivre ?47 Ces prostituées, mères symboliques, forment une famille autour du jeune enfant. Par la suite, c'est un veuf « cultivé, raffiné, de bon aspect ${ }^{48}$, « travailleur, honnête et paisible ${ }^{49}$, Mariano Salazar Montalvo, poussé à se refugier dans la maison close par une forte tempête, qui accepte de s'occuper de l'éducation du jeune enfant et de le considérer comme son propre fils : " cet enfant serait comme le sien, comme ce petit qui est mort en même temps que son épouse, sans lui donner le temps de connaître la paternité $»^{50}$; « il lui dit qu'il lui donnerait une éducation et qu'il ne manquerait jamais de rien. Il l'élèverait comme son fils $» .{ }^{11}$ Avec la domestique Tonalá, ils vont former pour le jeune enfant un couple de parents sociaux, répondant non seulement à ses besoins affectifs mais l'ouvrant également à la culture et à tous les savoirs (historiques, géographiques, littéraires et scientifiques). ${ }^{52}$ C'est ainsi que Gilberto deviendra le compagnon de travail du grand muraliste mexicain Diego Rivera.

Dans ce roman, tout laisse à penser que la relation entre Gilberto et Mariano Salazar Montalvo relève de la " possession d'état ", déjà connue par les droits romain et canonique. En effet, les trois principaux éléments permettant d'établir le rapport de filiation et de parenté entre le jeune homme et sa famille sont présents : le nomen (le nom) car Gilberto apprend à la fin de l'adolescence que Mariano lui a donné son nom, le tractatus (le traitement), "le fait de pourvoir [...] à l'entretien, à l'éducation et à l'installation de l'enfant » ${ }^{53}$ et la fama (la réputation), c'est-à-dire la reconnaissance de la situation de l'enfant par la société et la famille. ${ }^{54}$ Néanmoins, Gilberto n'est en aucun cas adopté du point de vue juridique, ni de manière simple, c'est-à-dire qu'il conserve des liens avec sa mère par le sang, ni a fortiori de manière plénière, en rupture avec sa famille d'origine. ${ }^{55}$ Bien qu'abandonnés ou complètement délaissés par leurs parents biologiques, Gilberto, Tita et ses neveux, ne sont jamais adoptés légalement par les adultes qui les recueillent et entretiennent avec eux de véritables relations affectives. Au regard du droit, l'ensemble des fonctions dévolues aux parents est interdite à ces personnages : s'ils pratiquent la parentalité, en s'occupant des soins physiques et psychiques de l'enfant, ils ne peuvent exercer la parentalité, être reconnus comme les parents légaux et inscrire ces enfants dans leur propre filiation. ${ }^{56}$ Leur parenté n'est ici que sociale, élective, mais nullement reconnue par le droit.

Dans le même ordre d'idée, il est intéressant de noter, dans Por debajo del agua, le comportement de Hugo qui, travesti en femme prénommée Isabel, se charge non seulement des nombreux accouchements de la soldadera Dolores, qui enfante dans la douleur ${ }^{57}$, mais considère tous les enfants se trouvant à la guerre avec leurs parents comme ses propres enfants ${ }^{5}$, ne rechignant pas à les porter sur le dos ${ }^{59}$, leur donnant à manger, leur racontant des histoires ${ }^{60}$, leur offrant même un sein plat et stérile pour les calmer. ${ }^{61}$

Ces dernières années, l'élargissement du mariage aux couples homosexuels vient souligner, de manière plus visible encore, combien l'idée de projet familial l'emporte sur le soi-disant « ordre symbolique » qui, de fait, légitime les discriminations en s'appuyant sur l'idée d'une nature -la différence des sexes- que la société devrait respecter afin d'éviter, selon certains de ses défenseurs les plus outranciers, de sombrer dans les ténèbres... L'idée qui y est rattachée -les homosexuels seraient des " fossoyeurs de l'humanité »- est complètement remise en cause dans le roman de Fernando Zamora. Le narrateur à la première personne n'est autre que le petit-fils biologique de la 
prostituée Silvette et de l'homosexuel Hugo, c'est-à-dire le descendant direct de l'union de deux êtres stigmatisés. ${ }^{62}$ La portée symbolique est forte ici et il n'est pas anodin que le roman se déroule dans un moment paradigmatique de l'histoire du pays, celui de la construction de l'image du « macho » mexicain qui persiste encore de nos jours. ${ }^{63}$ Cet enfant, élevé par son oncle, déconstruit non seulement le mythe de la famille traditionnelle et montre, si besoin était, qu'il n'est pas un monstre mais il rend compte également que les homosexuels ne commettent pas de « crime contre l'humanité » en annulant toute reproduction de l'espèce humaine. Il est intéressant de noter, à ce propos, que c'est le personnage le plus efféminé qui assure la descendance, tandis que celui qui rompt la chaîne généalogique, le Général Pablo Aguirre, incarne l'image par excellence du mâle mexicain. ${ }^{64}$ Le récit du narrateur s'inscrit dans une évidente volonté de dresser une généalogie, que l'on refuse aux homosexuels, et de montrer que le subalterne a la possibilité de " créer et de former de bons citoyens pour la société mexicaine ${ }^{65}$, comme le démontrent encore, dans Por si no te vuelvo a ver, les retrouvailles entre Gilberto et sa mère qui sont l'occasion d'intégrer cette dernière dans le groupe social privilégié auquel il appartient depuis son abandon et de redessiner une généalogie à partir d'une ancienne prostituée, pauvre et indienne.

L'écrivaine française Christine Angot expliquait récemment que « la littérature montre comment le réel tient $\gg .{ }^{66}$ Cette phrase est particulièrement signifiante à l'heure où beaucoup ne voient pas l'intérêt de ce champ. Pourtant, la littérature, en tant que chambre d'écho d'une époque, nous permet d'illustrer les changements opérés au sein de la société et, dans le cas précis des trois romans mexicains analysés ici, de montrer l'écart existant entre un modèle familial que l'on présuppose idéal et naturel et une réalité bien différente et beaucoup plus nuancée.

\section{Notes}

1 L'édition de référence est Laura Esquivel, Como agua para chocolate, Barcelone, Mondadori, 1994. Toutes les citations renvoient à cette édition. Les traductions françaises sont d'Eduardo Jiménez et Jacques Rémy-Zéphir dans Laura Esquivel, Chocolat amer, Paris, Gallimard, 2009.

2 Sur la réception critique de cette œuvre, lire Nicolas Balutet et Olga Chesnokova, " Como agua para chocolate de Laura Esquivel : recepción crítica y traductológica », e-scrita. Revista do Curso de Letras da UNIABEU, Nilópolis, vol. 4, nº1, 2013, pp. 213-230.

3 Esquivel, p. 56.

4 « infinidad de bofetadas ». Esquivel, p. 18.

5 « una bofetada fenomenal [que la hizo] rodar por el suelo ». Esquivel, p. 29.

6 « una paliza fenomenal, como nunca antes la había recibido ni la volvería a recibir ». Esquivel, p. 41.

7 Esquivel, p. 109.

8 « Mamá Elena era especialista en partir sandía: tomando un cuchillo filoso, encajaba la punta de tal manera que sólo penetraba hasta donde terminaba la parte verde de la cáscara, dejando sin tocar el corazón de la sandía. Hacía varios cortes en la cáscara de una perfección matemática tal, que cuando terminaba tomaba entre sus manos la sandía y le daba un solo golpe sobre una piedra, pero en el lugar exacto y mágicamente la cáscara de la sandía se abría como pétalos en flor, quedando sobre la mesa el corazón intacto. Indudablemente, tratándose de partir, desmantelar, desmembrar, desolar, destetar, desjarretar, desbaratar o desmadrar algo, Mamá Elena era una maestra ». 
Esquivel, pp. 87-88.

9 " no sólo podía partir costales y costales de nueces en pocos días, sino que gozaba enormemente practicando esta labor. Prensar, destrozar y despellejar eran algunas de sus actividades favoritas ». Esquivel, pp. 197-198.

10 Mamá Elena est castratrice. Il suffit pour s'en convaincre de rappeler le peu d'importance qu'ont les hommes dans le roman ou la figure de Pedro qui lui obéit sans maugréer, sans oublier les deux allusions explicites au pouvoir de castration du personnage : « on s'était trompé, c'était elle qu'il aurait fallu châtrer, comme ça on aurait eu au moins une vraie raison de lui refuser le mariage » [ " se había elegido mal al sujeto apropiado para capar, la adecuada era ella, de esta manera habría al menos una justificación real para que le estuviera negado el matrimonio ». Esquivel, p. 29] ; « durant l'enterrement, Tita versa des larmes sincères. Non pas sur la mère castratrice qui l'avait brimée toute sa vie, mais sur l'être séparé de celui qu'elle aimait » [« durante el entierro Tita realmente lloró por su madre. Pero no por la mujer castrante que la había reprimido toda la vida, sino por ese ser que había vivido un amor frustrado ». Esquivel, p. 122].

11 « con ella, había hecho una excepción, la había empezado a matar desde niña, poco a poquito ». Esquivel, p. 47.

12 « la imagen de una madre bruja sujetando las quijadas de sus hijos es la imagen de la desnutrición espiritual, de la censura de las palabras, del silencio de la poesía, de la mente encadenada. En pocas palabras, es la imagen de una verdadera hija de... Coatlicue, la devoradora » (ma traduction). Laura Esquivel, Íntimas suculencias. Tratado filosófico de cocina, Madrid, Ollero \& Ramos Editores, Plaza \& Janés Editores, 1998, pp. 151-152.

13 L'édition de référence est Fernando Zamora, Por debajo del agua, México, Plaza y Janés, 2002. Toutes les citations renvoient à cette édition. Les traductions françaises sont miennes.

14 L'édition de référence est Laura Martínez-Belli, Por si no te vuelvo a ver, Mexico, Planeta, 2008.Toutes les citations renvoient à cette édition. Les traductions françaises sont miennes.

15 Hervé Hamon, "Droit et parentalité », in Christian Robineau (dir.), Filiations à l'épreuve, Toulouse, ERES, 2002, p. 83.

16 « con un hombre de identidad desconocida ». Zamora, p. 18.

17 Zamora, pp. 15-16, 18.

18 « Je ne veux plus jamais te voir dans cette maison [...] Je ne veux plus te voir, ni toi ni personne. Je veux que vous me fichiez la paix. Que vous partiez et me laissiez en paix. - Et Daniel ? [...] - Prends-le avec toi ! Et pars avec lui n'importe où ! Je ne veux pas qu'il soit avec moi, je ne veux pas le voir non plus. - Tu nous vire ?, demande Carlos en colère. - Oui, oui si c'est comme cela que tu le vois, oui : tous. Vous êtes tous congédiés ! - Congédiés ? [...] Nous ne sommes pas tes employés! Tu ne peux pas nous congédier. Le père sourit. - Bien sûr que je le peux ! Tu es congédié, barre-toi maintenant ! " [ « No quiero que vuelvas a esta casa nunca más. [...] No quiero que vuelvas, ni tú ni nadie. Quiero que me dejen en paz. Que todos ustedes se larguen y me dejen en paz. - ¿Y Daniel? [...] -iLlévatelo! iAdonde sea! No quiero que esté conmigo, ni quiero verle la cara tampoco. - ¿Nos estás corriendo? - pregunta Carlos enojado. - Sí, si así quieres verlo, sí: a todos. iTodos están despedidos! - ¿Despedidos? [...] ¿No somos tus criados? No puedes despedirnos. El padre sonríe. - iClaro que puedo! iEstás despedido, así que lárgate ya! ». Zamora, p. 161].

19 " elle s'inquiétait de ne pas pouvoir offrir à son fils une vie meilleure, un environnement différent » [ "se preocupaba por no poder ofrecerle a su hijo una vida mejor, un ambiente distinto ». Martínez-Belli, p. 25] ; " elle demanda ensuite à cet homme qu'elle connaissait à peine, un de plus dans la liste infini des hommes de sa vie s'il avait la bonté d'éloigner cet enfant de la maison de passes, de lui enseigner tout ce qu'elle n'était pas capable de faire, et de donner au garçonnet [...] la chance qui lui était refusé avec elle » [«Después le pidió a aquel hombre al que apenas conocía, uno más en la infinita lista de hombres de su vida, que por favor se llevara a su hijo del burdel, que le enseñara todo lo que ella no era capaz y le diera al muchacho [...] la oportunidad que con ella le estaba negada ». Martínez-Belli, p. 34] ; « Elle savait qu'avec un seul mot de sa part, sa vie prendrait un tour bien différent. Elle se déferait de son fils, son chéri, la seule raison qui la maintenait en vie. Elle ne reverrait pas son Gilberto. Elle ne savait 
pas si elle aurait assez de forces pour supporter cette situation. Même si ce ne serait pas comme l'abandonner à son sort. Mariano lui ouvrirait une porte qui le mènerait à une vie meilleure. Il vivrait dans un endroit où cela ne sentirait pas la sueur, l'alcool, le sexe, le tabac ou le parfum » [ "Sabía que con una sola frase suya, su vida daría un vuelco tremendo. Se desprendería de su hijo, su adorado, la única razón por la que vivía. No volvería a ver a su Gilberto. No sabía si tendría fuerzas para soportarlo. Aunque no sería como abandonarlo a su suerte. Mariano le abriría una puerta que lo llevaría hacia otra vida mejor. Viviría en un lugar en donde no olería a sudor, ni a alcohol, a sexo, tabaco o perfumes ». Martínez-Belli, pp. 34-35].

20 « Elle l'embrassa tendrement sur les lèvres, sur le front rond et le serra contre sa poitrine » [ « Lo besó con amor en los labios, en la frente redonda y lo estrechó contra su pecho ». Martínez-Belli, p. 19] ; « à quelques mètres de distance, le bébé la suivait, son Gilberto, comme elle disait » [" a pocos metros de distancia, la seguía el niño, su Gilberto, como decía ella ». Martínez-Belli, p. 32] ; « elle fondit en larmes de remords en se rappelant comment elle essaya de se débarrasser de son enfant, qui s'était avéré si beau, si bon et intelligent » [ " rompió en lágrimas de remordimiento al recordar cómo intentó deshacerse de su chamaco, que le había salido tan guapo, tan bueno e inteligente ». Martínez-Belli, p. 33] ; "Elle savait que c'était mieux pour Gilberto, mais en même temps elle pensait que la vie perdrait son sens une fois que son petit serait parti. Mercedes lui caressa sa petite tête ronde, ses cheveux noirs de jais, ses douces oreilles de papier. Elle resta ainsi jusqu'à l'aube, le veillant, se réveillant en silence, essayant de contrôler sans succès la douleur incommensurable de la séparation » [« Sabía que era lo mejor para Gilberto, pero al mismo tiempo pensaba que la vida perdería sentido una vez que se fuera su pequeño. Mercedes le acarició la cabecita redonda, el pelo negro de alquitrán, las orejas suaves de papel. Estuvo así hasta que amaneció, velándolo, despidiéndose en silencio, intentando controlar sin éxito el dolor inconmensurable de la separación ». Martínez-Belli, p. 35] ; " elle l'embrassa fortement et le serra contre son ventre : souvenez-vous que vous serez toujours, toujours, dans mon cœur » [« ella lo abrazó con fuerza y lo estrechó contra su vientre : Recuerde que siempre, siempre, estará en mi corazón ». Martínez-Belli, p. 38] ; " quand elle pensait à Gilberto, elle estimait que c'était la seule épine qu'elle devait s'ôter avant de mourir. Il lui manquait tellement ! Il lui apparaissait en rêves, vêtu de ses culottes courtes, et elle le voyait comme si c'était hier » [ « cuando pensaba en Gilberto creía que ésa era la única espina clavada que debía sacarse antes de la muerte. iLo extrañaba tanto! Se le aparecía en sueños vestido con sus pantalones cortos, y lo veía como si fuera ayer ». Martínez-Belli, pp. 89-9o] ; «Une de ces nombreuses nuits, Mercedes se réveilla en sursaut, appelant Gilberto de ses cris. Malgré les années, les remords lui étaient encore douloureux. Quand elle se rendit compte qu'elle rêvait, elle posa ses mains sur son visage pour pleurer en silence » [ « Una de tantas noches, Mercedes despertó sobresaltada, llamando a gritos a Gilberto. A pesar de los años, aún le dolía el remordimiento. Cuando se dio cuenta de que soñaba, se llevó las manos a la cara para llorar en silencio ». MartínezBelli, p. 92].

21 Fernando Horcasitas Pimentel, La Llorona, Mexico, UNAM, 1950, p. 60 ; Fernando Horcasitas et Douglas Butterworth, «La Llorona », Tlalocan, vol. 4, nº 3,1963 , pp. 204-224 ; Luis González Obregón, Las calles de México. Leyendas y sucedidos,Mexico, Porrúa, 1997 ; p. 35 ; Artemio de Valle-Arizpe, Historia, tradiciones y leyendas de calles de México, Mexico, Diana, 1982, pp. 21-24.

22 Agnès Fine, "Le don d'enfant dans l'ancienne France », in Agnès Fine (dir.), Adoptions. Ethnologie des parentés choisies, Paris, Editions de la Maisons des sciences de l'homme, 1998, pp. 61-95.

23 Por debajo del agua est d'ailleurs considéré comme une prolongation tardive du «Roman de la Révolution ». Dans leur étude respective, Gabriel Osuna Osuna et María Rita Plancarte Martínez s'attardent sur les caractéristiques de ce courant littéraire que l'on retrouve dans l'œuvre de Fernando Zamora, par ailleurs souvent qualifiée de " premier roman gay de la Révolution » : narration littéraire de souvenirs personnels, expériences et chroniques autobiographiques du conflit armé, représentation de faits inspirés d'actions militaires et populaires qui ont permis des changements sociaux et politiques, faits décrits depuis le point de vue du peuple victime de la guerre, etc. Lire Gabriel Osuna Osuna, «Identidad y voz narrativa en Por debajo del agua de Fernando Zamora ", communication présentée lors du XXXVII Congreso Internacional del Instituto Internacional de Literatura Iberoamericana, Puebla, 26 juin 2008 ; et María Rita Plancarte Martínez, "Por debajo del agua de Fernando Zamora: el retorno al tema de la Revolución Mexicana ", Revista de literatura mexicana contemporánea, $\mathrm{n}^{\circ} 31$, 
2006, pp. 65-70.

24 Sur le féminisme dans l'œuvre de Laura Esquivel, lire Nicolas Balutet, « El poder de la comida en Como agua para chocolate. Acerca de la concepción esquiveliana del feminismo ", in Andjelka Pejović, Mirjana Sekulić et Vladimir Karanovićc (dir.), Comida y bebida en la lengua española, cultura y literaturas hispánicas, Kragujevac, FILUM, 2012, pp. 351-359.

25 Dans la Rome républicaine ou impériale par exemple, on distinguait le père ou la mère sociale, « ceux dont l'enfant porterait le nom ou hériterait par la suite, autrement dit le pater (le père) et la mater (la mère) ", de " ceux qui avaient participé à la conception biologique de l'enfant, autrement dit le genitor (le géniteur) et la genitrix (la génitrice) ». Lire à ce propos Laurent Barry, « Au fil des générations, entre biologie et anthropologie », in Noëlle Fiault (dir.), Identité(s), filiation, se repérer pour apprendre, Nice, SCEREN-CRDP de Nice, 2006, pp. 227-228.

26 Anne Lefebre-Teillard, "Filiation », in Denis Alland et Stéphane Rials (dir.), Dictionnaire de la culture juridique, Paris, PUF, 2003, p. 720.

27 Fondation Copernic, " Homosexualité, mariage et filiation ", Médiapart, 14 novembre 2012.

28 Irène Théry, Laurence Brunet, Jennifer Merchant et Martine Gross, "Filiation : l'impensé du projet Taubira », Le Monde, 18 septembre 2012. On sait que le mariage indissoluble n'est parvenu à être imposé par l'Eglise qu'au Moyen-Âge. Lire à ce propos Gabriel Langouët, Les « nouvelles » familles en France, Paris, Hachette, 1998 ; p. 15.

29 Cécile Deffontaines et Irène Théry, « Mariage homosexuel : "mariage et filiation se sont autonomisés" », Le Nouvel Observateur, 4 novembre 2012 ; Marcela Iacub, L'empire du ventre. Pour une autre histoire de la maternité, Paris, Fayard, 2004, pp. 41-52 ; Carmen García Mendieta, « La filiación: problemas jurídicos actuales », Anuario jurídico, vol. 13, 1986, p. 306.

30 Delphine de Mallevoüe, "Un test de paternité précoce contre l'infidélité », Le Figaro, 25 novembre 2011.

31 Esquivel, p. 122.

32 Caroline Mécary, "Homosexualité, mariage et filiation : où en sommes-nous ? ", Informations sociales, vol. 5, $\mathrm{n}^{\circ} 149,2008, \mathrm{p} .140$.

33 Martine Gross, " Mariage homosexuel : "Fonder la filiation sur l'engagement parental plutôt que sur la nature" », Le Monde, 5 février 2013.

34 Esquivel, p. 12.

35 Esquivel, p. 33.

36 « avec Nacha, elle retrouva les jeux de son enfance dans la cuisine, le marché, les galettes sortant du four, les noyaux colorés des chabanacos, les tortas de Noël, son chez-soi, l'odeur du lait bouilli, des petits pains au lait, du champurrado, du cumin, de l'ail, de l'oignon » [« ahí estaban, junto a Nacha, los juegos de su infancia en la cocina, las salidas al mercado, las tortillas recién cocidas, los huesitos de chabacano de colores, las tortas de Navidad, su casa, el olor a leche hervida, a pan de natas, a champurrado, a comino, a ajo, a cebolla ». Esquivel, p. 110] ; « elle regrettait ses odeurs de soupe au vermicelle, de chalaquiles, de champurrado, de sauce de malcajete, de pain au lait » [ « extrañaba su olor a sopa de fideos, a chilaquiles, a champurrado, a salsa de molcajete, a pan con natas ». Esquivel, p. 145].

37 « la imagen de Tonantzin-Guadalupe, la madre buena, la protectora, la que nutre, la que por medio de los alimentos libera el espíritu, la sensualidad y todos los deseos acumulados en silencio » (ma traduction). Laura Esquivel, Íntimas suculencias. Tratado filosófico de cocina, p. 152.

38 Esquivel, p. 13.

39 Esquivel, p. 145.

40 Esquivel, pp. 23, 31, 36.

41 Esquivel, p. 210.

42 A l'instar de Tita, Hugo Estrada, sous son identité d'Isabel, joue pendant la guerre le rôle de sage-femme : "Quand c'était au tour de Dolores d'accoucher (chose qui arrivait très souvent), c'était Isabel qui lui appliquait des cataplasmes et coupait le cordon ombilical » [" Cuando a Dolores le tocaba el turno de parir (cosa que sucedía muy a 
menudo) era Isabel quien le aplicaba fomentos y cortaba el cordón umbilical ». Zamora, p. 32] ; « Isabel coupe avec les dents le cordon du nouveau-né. Elle lui donne une petite tape sur les fesses. L'enfant se réveille enfin » [« Isabel corta con los dientes el cordón del recién nacido. Le da un pequeño golpe en el trasero. El niño despierta entonces ». Zamora, p. 42]. Elle fait montre également d'un grand «instinct maternel »: «Elle prend soin de l'enfant avec toute la tendresse qui lui reste. C'est ainsi qu'elle est partie vers le Nord, l'enfant dans les bras : toute l'après-midi et une bonne partie de la nuit, marchant et chantant entre les wagons pour que Salud ne pleure pas et, ensuite, pour qu'elle ne se réveille pas » [ « se hace cargo del niño con toda la ternura que le queda. Así se fue para el norte, con el niño en los brazos: toda la tarde y buena parte de la noche, paseando y cantando entre los vagones para que Salud no llorara y luego, para que no fuera a despertarse ». Zamora, p. 141].

43 « Tita, arrodillada frente a Rosaura, con gran desesperación pidió a Nacha que la iluminara en estos momentos. iSi era posible que le dictara algunas recetas de cocina, también era posible que le ayudara en este difícil trance! Alguien tenía que asistir a Rosaura desde el más allá, porque los del más acá no tenían manera. No supo por cuánto tiempo rezó de hinojos, pero cuando por fin despegó los párpados, el obscuro túnel de un momento a otro se transformó por completo en un río rojo, en un volcán impetuoso, en un desgarramiento de papel. La carne de su hermana se abría para dar paso a la vida. [...] Tomó al niño entre sus manos, se lo llevó a Rosaura, y juntas lloraron un rato, abrazadas a él. Después, siguiendo las instrucciones que Nacha le daba al oído, supo perfectamente todos los pasos que tenía que seguir : cortar el cordón umbilical en el lugar y momento preciso, limpiar el cuerpo del niño con aceite de almendras dulces, fajarle el ombligo y vestirlo. Sin ningún problema supo cómo ponerle primero la camiseta y la camisa, luego el fajero en el ombligo, luego el pañal de manta de cielo, luego el de ojo de pájaro, luego la franela para cubrirle las piernas, luego la chambrita, luego los calcetines y los zapatos y, por último, utilizando una cobija de felpa le cruzó las manos sobre el pecho para que no se fuera a rasguñar la cara. Cuando por la noche llegaron Mamá Elena y Chencha acompañada de los Lobo, se admiraron del profesional trabajo que Tita realizó. [...] El doctor Brown la examinó exhaustivamente. Fue entonces que supieron lo peligroso que había estado el parto. Según el doctor, Rosaura sufrió un ataque de eclampsia que la pudo haber matado. Se mostró muy sorprendido de que Tita la hubiera asistido con tanto aplomo y decisión en condiciones tan poco favorables. » Esquivel, pp. 68-69.

44 « Il cherchait désespérément son lait entre les seins de Tita. S’il y avait une chose dans la vie que Tita ne supportait pas, c'était de voir un affamé lui réclamer à manger sans pouvoir le satisfaire. Incapable de se contenir plus longtemps, elle ouvrit sa blouse et offrit sa poitrine à l'enfant. Elle la savait complètement sèche, mais son sein lui servirait au moins de tétine et l'occuperait tandis qu'elle s'efforçait de trouver une idée pour calmer sa faim. L'enfant happa le mamelon avec l'énergie du désespoir, et il téta avec une telle force qu'il parvint à extraire du lait de Tita. Quand celle-ci se rendit compte que l'enfant se détendait et qu'elle l'entendit déglutir, elle trouva ça bizarre. Elle nourrissait l'enfant ? Pour en avoir le cœur net, elle éloigna la petite bouche de sa poitrine et vit jaillir une giclée de lait. Tita était stupéfaite. Impossible qu'une célibataire ait du lait, il s'agissait d'un fait surnaturel et inexplicable ». [ " Buscaba desesperado su leche entre los senos de Tita. Si hay algo en la vida que Tita no resistía era que una persona hambrienta le pidiera comida y que ella no pudiera dársela. Le provocaba mucha angustia. Y sin poderse contener por más tiempo, Tita se abrió la blusa y le ofreció al niño su pecho. Sabía que estaba completamente seco, pero al menos le serviría de chupón y lo mantendría ocupado mientras ella decidía qué hacer para calmarle el hambre. El niño se pescó del pezón con desesperación y succionó y succionó, con fuerza tan descomunal que logró sacarle leche a Tita. Cuando ella vio que el niño recuperaba poco a poco la tranquilidad en su rostro y lo escuchó deglutir sospechó que algo extraño estaba pasando. ¿Sería posible que el niño se estuviera alimentando de ella? Para comprobarlo, separó al niño de su pecho y vio cómo le brotaba un chisguete de leche. Tita no alcanzaba a comprender lo que sucedía. No era posible que una mujer soltera tuviera leche, se trataba de un hecho sobrenatural y sin explicación en esos tiempos ». Esquivel, p. 71].

45 « Elle préférait nourrir Esperanza comme Nacha l'avait nourrie elle-même à sa naissance : avec atoles et infusions [...] elle poussait donc à grand renfort d'infusions et d'atoles dans ce paradis chaleureux rempli d'odeurs et de saveurs » [" prefirió en cambio proporcionarle a Esperanza la misma alimentación que Nacha había utilizado con ella cuando era una indefensa criatura : atoles y tés [...] así que con tés y atoles crecía de lo más sana entre los olores y los sabores de este paradisíaco y cálido lugar ». 
Esquivel, pp. 128-129].

46 « que lo mimaban y protegían como si se tratara de un gorrión que se ha dado de bruces contra un portón de vidrio. Le contaban cuentos por la noche, le enseñaban a vestirse, le hacían cosquillas en la panza y le permitían pintarrajear cuanto quisiera sobre las paredes de la cocina ». Martínez-Belli, p. 20.

47 Martínez-Belli, p. 26.

48 « culto, refinado, bien parecido ». Martínez-Belli, p. 77.

49 « trabajador, honrado y pacífico ». Martínez-Belli, p. 59.

50 « ese niño sería como suyo, como aquel pequeño que falleció junto a su esposa, sin darle tiempo de conocer la paternidad ». Martínez-Belli, p. 34.

51 « le dijo que no sólo le daría educación, sino que jamás le faltaría nada. Sería criado como un hijo ». Martínez-Belli, p. 35. Mariano a lui-même été élevé par son oncle et sa tante car son père est mort à la guerre et sa mère a été interné dans un asile psychiatrique. Martínez-Belli, pp. 60-67.

52 Martínez-Belli, pp. 42-43, 49.

53 Jacques Massip, Le nouveau droit de la filiation, Paris, Defrénois, 2006, p. 11.

54 Sur la possession d'état, lire Claire Neirinck, Maryline Bruggeman, Maïalen Contis et Laurence Monnier-Saillol, Droit de la famille, Paris, Ellipses, 2002, pp. 59-64 ; David Bakouche, Droit civil. Les personnes. La famille, Paris, Hachette, 2005, p. 207 ; et Nathalie Baillon-Wirtz, Yves Honhon, Marie-Christine Le Boursicot, Alice Meir-Bourdeau, Imran Omarjee et Clotilde Pons-Brunetti, L'enfant sujet de droits. Filiation, patrimoine, protection, Rueil-Malmaison, Lamy, 2010, p. 30.

55 Bakouche, pp. 201/247.

56 Hamon, p. 83.

57 « Si c'est au tour de Dolores d'accoucher, chose qui arrive fréquemment, c'est Isabel qui applique des cataplasmes, coupe le cordon ombilical avec les dents et noue le nombril du bébé avec une petite mèche de ses cheveux. Deux des neufs enfants que Dolores a eu durant la Révolution voyagent avec elles : Salud et Carmen, qui sont très fières d'être venues au monde dans les mains d'Isabel » [ « Si a Dolores le toca el turno de parir, cosa que sucede muy a menudo, es Isabel quien aplica fomentos, corta con los dientes el cordón umbilical y con un mechoncito de su trenza amarra el ombligo del bebé. Viajan con ellas dos de los nueve niños que tuvo Dolores en la Revolución : Salud y Carmen, quienes están bien orgullosos de haber llegado al mundo en manos de Isabel ». Zamora, p. 48] ; « Le moment venu, Isabel nettoie le sang et la merde et coupe avec les dents le cordon du nouveau-né. Elle lui donne un petit coup sur les fesses. L'enfant se réveille et goûte ce qu'est l'air... » [ "Llegado el momento, Isabel limpia la sangre y la mierda y corta con los dientes el cordón del recién nacido. Le da un pequeño golpe en el trasero. El niño despierta y sabe lo que es el aire... ». Zamora, p. 57].

58 « Isabel le voit, à l'instar de toues les enfants de la guerre, comme un de ses enfants, un parent » [ « Isabel lo considera, como a todos los muchachos de la guerra, un hijo suyo, un familiar ». Zamora, p. 48].

59 « La nostalgie d'Isabel prend également fin et elle étreint bien fort Carmen pour que la petite ne se casse pas la figure sur le sol » [ "Se rompe también la nostalgia de Isabel que abraza bien fuerte a Carmen para que la niña no se rompa las narices al suelo ». Zamora, p. 49] ; "Isabel a encore des forces pour porter Salud, le fils de Dolores » [« Isabel todavía tiene fuerzas para cargar a Salud, el hijo de Dolores ». Zamora, p. 53].

60 «Pour Isabel il n'y a rien de mieux que d'alimenter ces enfants et de leur raconter des histoires au coin du feu. Elle se sent si épanouie qu'elle n'essaye pas de rendre conscient ce bonheur » [ "Para Isabel no hay nada mejor que alimentar a estos niños y contar historias con ellos frente a la lumbre. Se siente tan completa que no tiene tentación de hacerse consciente de su felicidad ». Zamora, p. 54)] ; « En un instant, la soldadera lui prépare une décoction. Ils inventent des histoires, font la liste de tout ce que l'on peut voir dans les étoiles et jouent aux bois de Saint Jean » [« En un rato, la soldadera le prepara un cocimiento. Inventan cuentos, hacen lista de todo lo que puede verse en las estrellas y juegan a los maderos de San Juan ». Zamora, p. 59].

61 «Un peu en plaisantant, elle se blottit dans un coin du wagon, lève son haut et montre son téton au bébé. L'enfant mordille avec sa bouche sans dents mais l'instinct le rassure. Hugo se sent vide, comme son mamelon sans lait » [« Un poco en broma, se 
acurruca en la esquina del vagón, levanta las telas y le muestra al bebé su tetilla. El niño muerde con boca sin dientes pero el instinto lo tranquiliza. Hugo se siente vacío, como su tetilla sin leche ». Zamora, pp. 179-180].

62 " de cette relation dans le bordel de Margarita, de cette relation entre Hugo et Isabel, naquit un enfant, et cet enfant fut mon père " [ « de aquella relación en el burdel de Margarita, de la relación entre Hugo e Isabel, nació un niño, y ese niño fue mi padre ». Zamora, p. 171]. Lire également la scène du coït, Zamora, pp. 87-88.

63 Antoine Rodriguez, "Ser Leandro es contravenir las reglas de Dios: transtextualidad y transgenericidad en la novela Por debajo del agua de Fernando Zamora, México, 2002 », Lectures du genre, nº 6, 2009, p. 115.

64 Rodriguez, p. 118.

65 « crear y formar buenos ciudadanos para la sociedad mexicana ». Guadalupe PérezAnzaldo, «Por si no te vuelvo a ver : más allá de la imagen estigmatizada de la mujer pública en la literatura mexicana », Ámbitos Feministas, volume 2, nº 2, 2012 pp. 43-44.

66 Christine Angot, « Non, non, non, et non », Le Monde, 23 février 2013.

\section{Pour citer cet article}

Référence électronique

Nicolas BALUTET, "Filiations mexicaines au temps de la Révolution », Transtext(e)s

Transcultures 跨文本跨文化 [En ligne], 8 | 2013, mis en ligne le 02 décembre 2013,

consulté le 13 janvier 2014. URL : http://transtexts.revues.org/483

\section{Auteur}

\section{Nicolas BALUTET}

Nicolas BALUTET est actuellement PRCE d'espagnol à l'Université Jean Moulin - Lyon 3 et membre du groupe de recherche MARGE. Spécialiste des questions liées au genre et à l'homosexualité au Mexique et à Cuba, il est l'auteur de plusieurs ouvrages parmi lesquels Ecrire le sida (Lyon, Jacques André Editeur, 2010), Homosexualité et imaginaire sexuel chez les Aztèques (Oxford, Archaeopress, 2008) et Ars homoerótica. Escribir la homosexualidad en las letras hispánicas (Paris, Editions Publibook Université, 2006).

\section{Droits d'auteur}

() Tous droits réservés 